\title{
Galactic Cosmic Strings as Sources of Primary Antiprotons
}

\author{
Glenn D. Starkman and Tanmay Vachaspati \\ Physics Department, Case Western Reserve University \\ Cleveland $\mathrm{OH}$ 44106-7079.
}

(September 11, 2018)

\begin{abstract}
A possible signature of a class of superconducting cosmic strings trapped in the Milky Way plasma is the emission of low energy antiprotons due to baryon number violating processes on the string. We find the terrestrial flux and the energy spectrum of such antiprotons. Current observational bounds on the flux of low energy antiprotons place a lower bound on the string tension which is comparable to that given by the electroweak scale.
\end{abstract}

Typeset using REVTEX 
Current particle physics models and cosmology suggest that a number of cosmological phase transitions have occurred since the big bang. Depending on the topology of the symmetry breakings occurring at the phase transitions, lineal relics of the pre-phase transition universe may have survived until the present epoch. These "cosmic strings" in the present universe could be detected by their gravitational signatures [1] or by their emission of energetic particles [2] and $\gamma$-rays [3] if they are superheavy. If they are heavy and superconducting, they could be seen via their electromagnetic signatures [4.5]. In this article, we point out that the emission of antiprotons by some light superconducting strings that are trapped in the galactic plasma may yield yet another observable signature that would be relevant for ongoing and future searches (eg. the proposed AMS observatory) for cosmic ray antiprotons.

A number of hypothetical particle physics models containing superconducting strings have been constructed [4,6]. String superconductivity arises because charged fermions have zero modes on the strings which can propagate along the string. Here we shall only be interested in superconducting strings in which the charge carriers are ordinary quarks and hence also carry baryon number. In this case, the growth of electric current on the string is accompanied by the production of baryon number on the string. This can be seen directly by counting the number of quarks and leptons that are produced (but still confined to the string) when an electric field is applied along the string [⿴囗⿴囗十). The production of particles of charge $q$ due to an electric field of magnitude $E$ along the string is given by

$$
\frac{d^{2} N}{d t d l}=\frac{q E}{2 \pi}
$$

where, $N$ is the number of particles produced by the full length of string. As the charges of the electron and the $u$ and $d$ quarks are in the ratio $-3: 2:-1$, the numbers in which they are produced are in the ratio $-3: 3 \times 2:-3 \times 1$. (The quarks are thrice as numerous as the electrons because of their color degree of freedom; the minus sign means that antiparticles

\footnotetext{
${ }^{1}$ Our analysis only applies to strings on which there are quark and lepton zero modes.
} 
are produced.) It is easy to see that this production of particles is also accompanied by the production of baryon number. This follows since if 6 up quarks are produced then only 3 down antiquarks are produced. Each quark (antiquark) has baryon number $1 / 3(-1 / 3)$ and so the baryon number produced is $6 / 3-3 / 3=1$. If the electric field were applied in the opposite direction, antibaryons would be produced.

The application of an electric field along the string generates an electric current on the string which can grow until a critical current is reached. This critical current is given by

$$
i_{c}=\kappa(\alpha \mu)^{1 / 2}
$$

where $\kappa$ is a numerical constant that depends on the details of the particle physics, $\mu$ is the mass per unit length of the string and $\alpha$ is the fine structure constant. The existence of a critical current follows because when the fermions on the string occupy high enough energy levels, they can jump off the string into the ambient vacuum. So the critical current is roughly given by the mass of the fermions in vacuum $\left(i_{c} \sim m_{v a c}\right)$. If the fermions are confined, however, the correct mass to consider would be the lowest mass hadron. In fact, in our case, the Fermi levels can only decrease by the leakage of baryons and so the correct mass for us to consider is the mass of the proton $\sim 1 \mathrm{GeV}$. With $\mu=m_{W}^{2} \mu_{-6} \mathrm{GeV}^{2}$ where $m_{W} \sim 100 \mathrm{GeV}$ is the mass of the $W$ boson and $\mu_{-6}$ is a free parameter, we find $\kappa \sim$ $0.1 \mu_{-6}^{-1 / 2}$. Note that $\mu_{-6}=1$ corresponds to strings formed at the electroweak scale with $\mu \simeq 10^{4} \mathrm{GeV}^{2} \simeq 10^{-6} \mathrm{~g} / \mathrm{cm}$

The dynamics of light superconducting cosmic strings has been investigated in a series of papers by Vilenkin and collaborators [5,4,1] leading to the following scenario. A cosmological phase transition can lead to the formation of a network of light, superconducting strings and the strings can be expected to be produced with small electric currents on them. (If there is a primordial magnetic field present, the electric currents can be much stronger.) Such a network of strings will be frozen in the cosmological plasma in a way similar to primordial magnetic fields. Once galaxy formation starts the strings flow into the protogalaxy together with the ambient plasma. Subsequent turbulence in the galactic flow stretches the strings 
and generates a tangle. The galactic turbulence drives the string energy to smaller and smaller length scales (higher curvature). But, at a critical curvature radius, $R_{*}$, the frozenin condition breaks down and the curved string breaks away from the ambient plasma and collapses.

There are two regimes that we need to consider depending on if $R_{*}$ is larger or smaller than the typical length scale $\left(L \sim 10^{20} \mathrm{~cm}\right)$ associated with galactic turbulence. On scales smaller than $L$, the plasma velocity is given by magnetohydrodynamic turbulence [12]:

$$
v_{R} \sim v_{L}\left(\frac{R}{L}\right)^{1 / 4}, \quad(R<L)
$$

while, on scales larger than $L$ the velocity is described by the Kolmogorov spectrum:

$$
v_{R} \sim v_{L}\left(\frac{R}{L}\right)^{1 / 3}, \quad(R>L)
$$

where $v_{L} \sim 10^{6} \mathrm{~cm} / \mathrm{s}$. The string curvature scale $\left(R_{*}\right)$ at which free collapse occurs can be found by equating the typical string velocity at that scale [7]

$$
v_{\text {string }} \sim \frac{c}{\kappa R} \sqrt{\frac{\mu}{\alpha \rho}}
$$

to the plasma velocity at the same scale. Here $\rho$ is the plasma density which we take to be $10^{-25} \mathrm{~g} / \mathrm{cm}^{3}$. For $R_{*}$ smaller than $L$, this gives [5]

$$
R_{*} \sim 10^{16} \kappa^{-4 / 5} \mu_{-6}^{2 / 5} \mathrm{~cm}, \quad\left(R_{*}<L\right)
$$

where, $\mu$ has been written as $10^{-6} \mu_{-6} \mathrm{~g} / \mathrm{cm}$. For $R_{*}$ larger than $L$, we get

$$
R_{*} \sim 10^{16} \kappa^{-3 / 4} \mu_{-6}^{3 / 8} \mathrm{~cm}, \quad\left(R_{*}>L\right)
$$

These calculations are valid only as long as $R_{*}$ is less than the size of the galaxy, $R_{g} \sim 10$ kpc. For $\kappa \sim 1$, this means that our calculations are valid for strings formed at scales below $\sim 10^{10} \mathrm{GeV}$. Heavier strings will not be trapped by the galactic plasma and will have different dynamics. 
The dynamics of the string during collapse is friction dominated and the terminal velocity is given by the turbulent velocity at the scale $R_{*}$ [7]:

$$
v_{*} \sim v_{L}\left(\frac{R_{*}}{L}\right)^{1 / 4}, \quad\left(R_{*}<L\right)
$$

Plugging in numerical values, we find

$$
v_{*} \sim 10^{5} \kappa^{-1 / 5} \mu_{-6}^{1 / 10} \mathrm{~cm} / \mathrm{s}, \quad\left(R_{*}<L\right)
$$

Similarly, for the Kolmogorov case we get

$$
v_{*} \sim 10^{5} \kappa^{-1 / 4} \mu_{-6}^{1 / 8} \mathrm{~cm} / \mathrm{s}, \quad\left(R_{*}>L\right) .
$$

The energy of the loop during collapse will be transformed into thermal energy of the surrounding medium. In the very final throes of its collapse, when the size of the loop is comparable to the thickness of the string, the loop will annihilate into the vacuum and release its remaining energy into various particles. (This last stage does not seem to be of much consequence since only a very tiny fraction of the string energy can be released this way.)

We now wish to find the baryon number released by the string network during its evolution. While a certain section of string is frozen-in, there is no electric field along it, and baryon number is not produced. But once the string section becomes curved on a scale smaller than $R_{*}$, it collapses under its own tension and, in doing so, cuts across the galactic magnetic field. By Faraday's law, the traversal across the magnetic flux is equivalent to an applied electric field along the string and hence will generate a current and the accompanying baryon number. The production of baryon number is independent of the details of the collapse - it only depends on the magnetic flux, $\Phi$, that the string cuts across. To see this we first find the electric field along the string:

$$
|\vec{E}|=|\vec{v} \times \vec{B}|
$$

where $\vec{v}$ is the velocity of the string. Inserting this expression in (1) yields the total number of particles produced during the collapse of a loop: 


$$
N=\frac{q}{2 \pi} \int d t \oint d l|\vec{v} \times \vec{B}|=\frac{q \Phi}{2 \pi}
$$

The magnetic flux $\Phi$ through a loop of size $R_{*}$ will be estimated by $B_{g} R_{*}^{2}$ where $B_{g}=$ $10^{-6} B_{-6} \mathrm{G}$ is the strength of the galactic magnetic field. Therefore the baryon number produced, $Q_{B}$, can be estimated as

$$
Q_{B} \sim \pm 0.1|\Phi| \sim \pm 0.1 B_{g} R_{*}^{2}
$$

Inserting numerical values gives:]:

$$
\begin{gathered}
Q_{B} \sim \pm 10^{30} \kappa^{-8 / 5} \mu_{-6}^{4 / 5} B_{-6}, \quad\left(R_{*}<L\right) \\
Q_{B} \sim \pm 10^{30} \kappa^{-3 / 2} \mu_{-6}^{3 / 4} B_{-6}, \quad\left(R_{*}>L\right) .
\end{gathered}
$$

The sign of the baryon number can be positive or negative depending on the orientation of the string with respect to the galactic magnetic field. We expect that both baryons and antibaryons will be produced in roughly equal numbers but in different regions of the galaxy.

The time scale on which a string loop of size $R_{*}$ collapses is

$$
t_{*}=R_{*} / v_{*} \sim 10^{11} \kappa^{-3 / 5} \mu_{-6}^{3 / 10} \mathrm{~s}, \quad\left(R_{*}<L\right)
$$

and,

$$
t_{*}=R_{*} / v_{*} \sim 10^{11} \kappa^{-1 / 2} \mu_{-6}^{1 / 4} \mathrm{~s}, \quad\left(R_{*}>L\right)
$$

To find the number density of antiprotons in our galaxy today we must sum up the number of antiprotons produced by all the loops over the entire lifetime of the galaxy $t_{g} \sim 10^{17} \mathrm{~s}$. This gives

$$
n_{\bar{p}} \sim \frac{\left|Q_{B}\right|}{R_{*}^{3}} \frac{t_{g}}{t_{*}}
$$

\footnotetext{
${ }^{2} \mathrm{~A}$ convenient conversion factor is $1 \mathrm{G}-\mathrm{cm}^{2}=3 \times 10^{4}$.
} 
The antiproton number density can be converted to a terrestrial energy flux in antiprotons by multiplying by the velocity of the antiprotons (order $c$ ). This yields:

$$
\begin{aligned}
& f_{\bar{p}} \sim 10^{2} \kappa^{7 / 5} \mu_{-6}^{-7 / 10} B_{-6} \mathrm{GeV} / \mathrm{m}^{2}-\mathrm{s}, \quad\left(R_{*}<L\right) \\
& f_{\bar{p}} \sim 10^{2} \kappa^{5 / 4} \mu_{-6}^{-5 / 8} B_{-6} \mathrm{GeV} / \mathrm{m}^{2}-\mathrm{s}, \quad\left(R_{*}>L\right) .
\end{aligned}
$$

The dependence of these estimates on the string scale are more transparent in the special case when only quark and lepton zero modes are present. Then we can use use $\kappa \sim 0.1 \mu_{-6}^{-1 / 2}$ and the estimates become

$$
\begin{gathered}
f_{\bar{p}} \sim 10 \mu_{-6}^{-7 / 5} B_{-6} \mathrm{GeV} / \mathrm{m}^{2}-\mathrm{s}, \quad\left(R_{*}<L\right) \\
f_{\bar{p}} \sim 10 \mu_{-6}^{-5 / 4} B_{-6} \mathrm{GeV} / \mathrm{m}^{2}-\mathrm{s}, \quad\left(R_{*}>L\right) .
\end{gathered}
$$

Note that the antiproton flux decreases with increasing string tension. So heavier strings produce fewer antiprotons and the largest flux of antiprotons is due to the lightest strings. Assuming that the standard model is correct up to the electroweak scale we have $\mu_{-6}>1$ and this gives us an upper bound on the antiproton flux from strings.

These estimates assume that the antiprotons do not leak out of the galaxy volume and neither are they annihilated in scatterings off protons. Both assumptions can be justified by rough estimates. The leakage timescale, $\tau_{\text {leak }}$, is found as the time taken by a relativistic antiproton (speed $\sim c$ ) to cover a distance equal to the size of the galaxy $L_{g} \sim 10 \mathrm{kpc}$. A crucial factor that needs to be accounted for is that the antiproton trajectory is not a straight line but piecewise circular due to the galactic magnetic field. Assuming a random walk for the antiproton with step size similar to the cyclotron radius $\xi \sim m c / B_{g}$ we find

$$
\tau_{\text {leak }} \sim \frac{L_{g}^{2}}{\xi} \sim 10^{19} \mathrm{~s}
$$

which is longer than the age of the galaxy. The scattering timescale, $\tau_{\text {scatt }}$, of the antiprotons is found by using the proton-antiproton scattering crosssection $\sigma_{p \bar{p}} \sim m_{p}^{-2}$ where $m_{p}$ is the 
mass of the proton and the galactic proton density $\sim 10^{-24} \mathrm{~g} / \mathrm{cm}^{3}$. This gives

$$
\tau_{\text {scatt }} \sim \frac{m_{p}}{\rho \sigma_{p \bar{p}}} \sim 10^{18} \mathrm{~s}
$$

and so the survival time for the antiprotons is longer than the age of the galaxy.

So far we have only considered the total flux of antiprotons. We now turn to the antiproton energy distribution. The antiprotons that come off the string due to $u$ and $d$ quarks on the string will have energy of order the proton mass. We might think that the $c$ and $s$ quarks will come off at energies when they can combine into baryons and that the $t$ and $b$ quarks can only jump off the string at energies greater than the top quark mass $(\sim 200$ $\mathrm{GeV}$ ). If true, the string would emit a third of the baryons at energies of a few hundred $\mathrm{GeV}$ which would then decay into protons and antiprotons of similar energies. This would be quite a distinctive signature for strings. However, this expectation ignores flavor changing processes by which $t$ quarks living on the string, scattering off $d$ quarks living on the string, can be emitted as $d$ quarks and $u$ quarks living off the string. The relevant terms in the Lagrangian for such an interaction are

$$
\frac{g}{2} U_{t d} \bar{d}_{L}^{(1)} \gamma^{\mu} t_{L}^{(0)} W_{\mu}^{-}, \quad \frac{g}{2} U_{d u}^{\star} \bar{u}_{L}^{(1)} \gamma^{\mu} d_{L}^{(0)} W_{\mu}^{+}
$$

where the superscripts refer to whether the fermionic field is a zero mode $(0)$ or a massive mode (1) and the quantity $U_{i j}$ is the $i j$ component of the CKM matrix (see [8] for example). So the interaction rate is suppressed due to the factor $\left|U_{t d}\right|^{2} \sim 10^{-4}$. In addition, there is a geometric suppression since only a fraction of the $d_{L}^{(1)}$ wavefunction overlaps with the $\bar{t}_{L}^{(0)}$ zero-mode wavefunction. This amounts to a suppression by $\left(m_{d} / m_{t}\right)^{2}$ in the cross-section; a less important final-state wave-function overlap is estimated as $\left(m_{u} / m_{d}\right)^{2}$. Combining these factors with the Fermi cross-section, and a quark number density on the string, $n_{q} \simeq p_{F} m_{q}^{2}$, gives a time scale for flavor changing interactions:

$$
\tau_{\text {flavor }} \sim\left[\frac{G_{F}^{2} E^{2}}{4 \pi^{2}}\left|U_{t d}\right|^{2}\left|U_{d u}\right|^{2}\left(\frac{m_{d}}{m_{t}}\right)^{2}\left(\frac{m_{u}}{m_{d}}\right)^{2}\left(p_{F} m_{d}^{2}\right)\right]^{-1} \sim 10^{3-4} \mathrm{~s}
$$

Actually, of course, the light quarks come off the string not as bare quarks, but as pions, with 
the baryon number remaining on the string. Thus, for example, $t^{(0)}+d^{(0)} \longrightarrow u^{(0)}+d^{(0)}+2 \pi^{0}$. We have neglected the QCD corrections to the final state.

This flavor changing rate is somewhat faster than the astrophysical rate associated with the build up of ( $1 \mathrm{GeV}$ of) Fermi momentum on the string as the string cuts through the galactic magnetic field:

$$
\tau_{\text {Fermi }} \sim \frac{1 G e V}{\dot{p}_{F}} \sim \frac{1 G e V}{q B v} \sim \frac{10^{11} \mathrm{~cm}}{q B_{-6} v}
$$

Using (8) and (9) this gives,

$$
\begin{gathered}
\tau_{\text {Fermi }} \sim 10^{6} \kappa^{1 / 5} \mu_{-6}^{-1 / 10} \mathrm{~s}, \quad\left(R_{*}<L\right) \\
\tau_{\text {Fermi }} \sim 10^{6} \kappa^{1 / 4} \mu_{-6}^{-1 / 8} \mathrm{~s}, \quad\left(R_{*}>L\right)
\end{gathered}
$$

This implies that the $t$ quarks will convert to $d$ quarks and escape from the string as $d$ quarks and the threshold for this to happen is $\sim 1 \mathrm{GeV}$ (instead of $\sim 174 \mathrm{GeV}$ ). Therefore, the Fermi momentum on the string will saturate at about $1 \mathrm{GeV}$ and only mildly relativistic protons and antiprotons will be emitted from the string].

Once the antiprotons are ejected from the string, we expect them to undergo acceleration by the usual mechanism of shock acceleration proposed by Fermi [9, [10]. This would lead to a spectrum of antiprotons whose energy distribution falls off as $E^{-2}$ and which is normalized by the total flux given in (20) and (21). If the antiproton energy flux at energy $E$ is denoted by $F(E)$, we have

$$
d F=f_{\bar{p}} \frac{m_{p}}{E} \frac{d E}{E}
$$

where $f_{\bar{p}}$ is given in eqns. (18) and (19) and $m_{p}$ is the mass of the proton.

The currently favored explanation for the observed antiprotons in cosmic rays is that they are produced as secondary particles due to cosmic ray collisions off interstellar matter

\footnotetext{
${ }^{3}$ For large $\mu_{-6}$ it may happen that $\tau_{\text {Fermi }}<\tau_{\text {flavor }}$ but then the total flux of antiprotons, as given by eqns. (20) and (21), is too small to be of interest.
} 
and present observations are consistent with this scenario (see [11] for a review and [13] for recent observations of low energy antiprotons). If we assume that the antiprotons seen so far are all secondary particles, then the current observations place an upper bound on the primary flux. The present bound for the antiproton flux in the kinetic energy range between 1 to $4 \mathrm{GeV}$ is $d F<10 \mathrm{GeV} / \mathrm{m}^{2}-\mathrm{s}$. So the antiproton flux from galactic superconducting strings produced at scales larger than the electroweak scale is not constrained by present observations. But continued observations and the proposed AntiMatter Search Observatory will either produce positive evidence for this possible exotic constituent of our Milky Way or impose interesting constraints.

\section{ACKNOWLEDGMENTS}

We are grateful to Tom Gaisser and Dick Mewaldt for advice and comments. 


\section{REFERENCES}

[1] A. Vilenkin and E.P.S. Shellard, Cosmic Strings and other Topological Defects (Cambridge University Press, Cambridge, 1994).

[2] P. Bhattacharjee, C.T. Hill and D.N. Schramm, Phys. Rev. Lett. 69, 567 (1992).

[3] M. Mohazzab and R. Brandenberger, Int. J. Mod. Phys. D2, 183 (1993).

[4] E. Witten, Nucl. Phys. B249, 557 (1985).

[5] E.M. Chudnovsky, G.B. Field, D.N. Spergel and A. Vilenkin, Phys. Rev. D34, 944 (1986).

[6] G. Lazarides, C. Panagiotakopoulos and Q. Shafi, Phys. Rev. Lett. 56, 432 (1987); Phys. Lett. B183, 289 (1987).

[7] E. Chudnovsky and A. Vilenkin, Phys. Rev. Lett. 61, 1043 (1988).

[8] T. P. Cheng and L. F. Li, Gauge Theory of Elementary Particle Physics (Oxford University Press, 1991).

[9] E. Fermi, Phys. Rev. 75, 1169 (1949).

[10] T.K. Gaisser, Cosmic Rays and Particle Physics (Cambridge University Press, Cambridge, 1990).

[11] T.K. Gaisser and R.K. Schaefer, Ap. J. 394, 174 (1992).

[12] Ya.B. Zel'dovich, A.A. Ruzmaikin and D.D. Sokoloff, Magnetic Fields in Astrophysics (Gordon and Breach, New York, 1983).

[13] K. Yoshimura et al., Phys. Rev. Lett. 75, 3792 (1995). 